\title{
Prognostic factors affecting early colectomy in patients with moderate to severe ulcerative colitis treated with calcineurin inhibitors
}

\author{
HIROMITSU BAN ${ }^{1}$, SHIGEKI BAMBA ${ }^{2}$, ATSUSHI NISHIDA ${ }^{2}$, OSAMU INATOMI ${ }^{2}$, MAKOTO SHIOYA ${ }^{2}$, \\ KEN-ICHIRO TAKAHASHI ${ }^{2}$, HIROTSUGU IMAEDA ${ }^{2}$, MASAKI MURATA ${ }^{2}$, \\ MASAYA SASAKI ${ }^{3}$, TOMOYUKI TSUJIKAWA ${ }^{4}$ and AKIRA ANDOH ${ }^{2}$
}

Divisions of ${ }^{1}$ Endoscopy, ${ }^{2}$ Gastroenterology and ${ }^{3}$ Clinical Nutrition; ${ }^{4}$ Department of Comprehensive Internal Medicine, Shiga University of Medical Science, Ōtsu, Shiga 520-2192, Japan

Received March 29, 2015; Accepted April 29, 2016

DOI: $10.3892 / \mathrm{etm} .2016 .3341$

\begin{abstract}
Calcineurin inhibitors (CNIs) such as cyclosporine A (CSA) and tacrolimus (FK506) are often used as a second-line drug for steroid-refractory or steroid-dependent patients with ulcerative colitis (UC). The aim of the present study was to determine the prognostic factors for early colectomy. A total of 85 hospitalized patients with UC (CSA, 50 patients; FK506,35 patients) were enrolled. Colectomy carried out within 60 days of starting CNI therapy was defined as 'early colectomy'. To assess the prognostic factors affecting early colectomy, clinical practical variables, including the Onodera-prognostic nutritional index (O-PNI): 10xAlb+0.005x (total lymphocyte count), were analyzed. The results demonstrated that the significant factors predicting early colectomy were i) disease severity, ii) immunomodulator-naïve history, iii) lower serum hematocrit, iv) lower serum albumin and v) lower O-PNI. In addition, the significant factors predicting overall colectomy were as follows: i) C7-HRP positivity and ii) $>10,000 \mathrm{mg}$ of prednisolone used prior to the initiation of CNI treatment. The combination of hematocrit and O-PNI enhanced the prediction of early colectomy. Clinical variables such as hematocrit and O-PNI were the significant factors predicting colectomy. These results may be used as a guide to predict the outcome of patients with UC in clinical settings.
\end{abstract}

\section{Introduction}

Ulcerative colitis (UC) is characterized by chronic enteritis, which is confined to the colon with remissions and exacerbations (1). Patients who do not respond to 5-aminosalicylic acid

Correspondence to: Dr Shigeki Bamba, Division of Gastroenterology, Shiga University of Medical Science, Seta-Tsukinowa, Ōtsu, Shiga 520-2192, Japan

E-mail: sb@belle.shiga-med.ac.jp

Key words: ulcerative colitis, colectomy, calcineurin inhibitors, cyclosporine A, tacrolimus compounds and corticosteroids are usually considered for treatment with calcineurin inhibitors (CNIs) or drugs such as infliximab and adalimumab (2).

CNIs, cyclosporine A (CSA) and tacrolimus (FK506), are effective in patients with steroid-refractory or steroid-dependent UC $(3,4)$. Although drugs such as infliximab and adalimumab are also used as rescue therapies in patients with refractory UC (5); practice guidelines do not indicate which treatment is preferable (5-9). However, in some studies, CSA has been demonstrated to provide better outcomes in patients with severe UC $(10,11)$ or in patients without cytomegalovirus reactivation (12-15). Therefore, CNIs are the preferred form of second-line treatment for steroid-refractory UC patients. Generally, patients who do not respond to CNIs are recommended to undergo colectomy (2). Third-line medical therapy may be considered only at a specialist centre (16). Therefore, certain patients undergo colectomy if the second-line therapy fails. In this context, it is important that the prognosis of the patient be established prior to starting the second-line therapy. The choice of the medical treatment or the intensity of the therapy may then be adapted according to its predicting factors.

The prognosis of UC has previously been investigated. Reinisch et al (17) demonstrated that newly-diagnosed UC patients with poor prognoses tended to be young non-smokers with high levels of inflammatory biomarkers, low levels of hemoglobin, and extensive disease based on colonoscopy testing. A population-based study also revealed that extensive colitis, a high number of systemic symptoms and a young age at diagnosis were factors associated with a high risk of colectomy (18). However, these results did not reflect severe conditions such as moderate or severe UC in patients who may require colectomy if treatment fails. Saito et al (19) demonstrated that age, platelet count, Lichtiger score and total protein could predict the clinical outcomes of cyclosporine treatment. However, the factors contained independent variables, which would become evident only after cyclosporine treatment had begun.

Our previous study demonstrated that the factors affecting the rate of colectomy at 1 year and the overall colectomy rate were i) CNI non-responsiveness, ii) $>10,000 \mathrm{mg}$ prednisolone used prior to the initiation of CNI treatment and iii) positivity 
for C7-horseradish peroxidase (HRP) (20). Based on these findings, the present study aimed to determine the factors that influence colectomy within 60 days of beginning treatment with CNIs using the Onodera-prognostic nutritional index (O-PNI). The O-PNI was originally used to predict the prognosis in 189 patients who underwent surgery of the gastrointestinal tract (21), and the equation used the serum albumin and total lymphocyte count. O-PNI has been applied for patients with end-stage liver disease, active tuberculosis, peritoneal dialysis, and with gastrointestinal malignancies (21-26).

The aim of the present study was to determine the prognostic factors for early colectomy following comparison with the results of our previous report (20), which would demonstrate the factors affecting the rate of colectomy at 1 year as well as the overall colectomy rate.

\section{Patients and methods}

Patients. We analyzed the medical charts and recent follow-up visits of 85 patients hospitalized with UC (treated with CSA, 50 patients; treated with FK506, 35 patients) who had been treated with CNIs as a second-line therapy for disease flare-ups between December 1999 and July 2014 at the Shiga University of Medical Science Hospital (Ōtsu, Japan; Table I). UC was purchased from Novartis (Tokyo, Japan) and FK506 was purchased from Astellas Pharma, Inc., (Tokyo, Japan). The clinical data were measured 3 days prior to the beginning of treatment with CNIs. The O-PNI was calculated based on the serum albumin and total lymphocyte count, using the following equation: $\mathrm{O}-\mathrm{PNI}=10 \mathrm{x}$ [serum albumin $(\mathrm{g} / \mathrm{dl})+0.005 \mathrm{x}$ total lymphocyte count $(/ \mathrm{ml})]$. Total lymphocyte count was obtained by multiplying the percentages of lymphocytes in the white blood cell count. CNIs were administered to the patients resistant to systemic corticosteroids (27). Prior to July 2009 when FK506 was approved for use under the National Health Insurance in Japan, CSA was the only choice for treatment. Cytomegalovirus reactivations were validated by cytomegalovirus antigenemia (C7-HRP). C7-HRP was measured by SRL Inc., which is a third party organisation independent of the study physician. All patients received concurrent ganciclovir (Mitsubishi Tanabe Pharma, Osaka, Japan) treatment due to cytomegalovirus reactivations. Ganciclovir was administered intravenously at a dose of $10 \mathrm{mg} / \mathrm{kg} / \mathrm{day}$, divided into two portions for two weeks. The present study was approved by the Ethics Committee of Shiga University of Medical Science.

CNI administration. The method for CSA administration has been previously described (27). Initial oral FK506 treatment was a dose of $0.1 \mathrm{mg} / \mathrm{kg} / \mathrm{day}$, divided into two portions. Blood was harvested for the determination of FK506 concentrations in the whole blood. Dosage was adjusted in order to maintain whole blood concentrations. Serum trough levels of FK506 were measured three times/week during admission. The dose was managed by first aiming for a high trough level of 10-15 ng/ml, which was calculated according to the following formula: Adjusted dose = previous dose x 12.5/(trough FK506 concentration). After 2 weeks of achieving high trough levels, the dose was reduced by aiming for a low trough level of $5-10 \mathrm{ng} / \mathrm{ml}$ for the following week, which was calculated according to the following formula: Adjusted dose $=$ previous
Table I. Clinical background of the patients.

Clinical characteristic

Result

Total no. of patients $(\mathrm{M} / \mathrm{F})$

$85(52 / 33)$

Age [years (range)]

$34.6(16-69)$

Disease duration [months (range)]

$58.4(0.3-267.7)$

Seo index (no. of patients $<220 / \geq 220$ )

$45 / 40$

Disease severity (no. of patients with

$29 / 56$

left-sided colitis/pancolitis)

Disease course (no. of patients with

$9 / 23 / 53$

first attack/chronic continuous/

relapse remitting UC)

Previous treatment (no. of patients

treated with cytapheresis therapy/

AZA or 6-MP/biologics)

Hematocrit [mean \% (range)]

White blood cell count $/ \mathrm{mm}^{3}$

[mean (range)]

Lymphocyte count $/ \mathrm{mm}^{3}$

[mean (range)]

Platelets $\left[\times 10^{3} / \mathrm{mm}^{3}\right.$; mean (range)

$341.2(44-933)$

Albumin g/dl [mean (range)]

$3.3(1.8-4.5)$

C-reactive protein $\mathrm{mg} / \mathrm{dl}$

$2.7(0.0-16.6)$

[mean (range)]

O-PNI [mean (range)]

$39.4(22.7-53.1)$

C7-HRP (no. of patients with

$23 / 62$

positive/negative C7-HRP)

PSL $\geq 10,000 \mathrm{mg}$ (no. of patients

$12 / 73$

with/without PSL $\geq 10,000 \mathrm{mg}$ )

Left-sided colitis refers to patients who have mucosal lesions restricted to the rectum, sigmoid colon and descending colon. $\mathrm{M}$, male; F, female; AZA, azathioprine; 6-MP, 6-mercaptopurine; O-PNI, onodera-prognostic nutritional index; C7-HRP, cytomegalovirus antigenemia; PSL, prednisolone.

dose x 7.5/(trough FK506 concentration). The absorption of FK506 may decrease by $38 \%$ following meals. To minimize the effect of meals, FK506 was prescribed to be taken 1 hour before meals.

Definitions. Disease activity was assessed using Seo's complex integrated disease activity index (28). Scores $>220$ were classified as severe disease. Moderate disease activity was defined as a score of 150-220. Scores $<150$ were classified as 'remission'. Patients were described as having an immunomodulator-naïve history if they did not have a history of immunomodulatiory medication. 'CNI responders' were defined as those with a 50 point score decrease during CNI administrations. 'Early colectomy' was defined as patients who had a colectomy procedure within 60 days of starting CNIs.

Statistical analysis. The medical backgrounds of the patients in the early colectomy group were compared with those of the colectomy-free group to determine the characteristics of early colectomy. Statistical differences were determined using 
Table II. Comparison of patient clinical backgrounds between the early colectomy group and the non-early colectomy group.

\begin{tabular}{|c|c|c|c|}
\hline Clinical characteristic & $\begin{array}{l}\text { Early } \\
\text { colectomy group }\end{array}$ & $\begin{array}{l}\text { Non-early } \\
\text { colectomy group }\end{array}$ & P-value \\
\hline No. of patients $(\mathrm{M} / \mathrm{F})$ & $12(8 / 4)$ & $73(44 / 29)$ & 0.6737 \\
\hline Age (mean; years) & 35.7 & 34.4 & 0.7181 \\
\hline Disease duration (mean; months) & 37.8 & 61.8 & 0.1770 \\
\hline Seo index (no. of patients $<220 / \geq 220$ ) & $2 / 10$ & $43 / 30$ & 0.0066 \\
\hline Disease severity (no. of patients with left-sided colitis/pancolitis) & $2 / 10$ & $27 / 46$ & 0.1689 \\
\hline $\begin{array}{l}\text { Disease course (no. of patients with first attack/chronic } \\
\text { continuous/relapse remitting ulcerative colitis) }\end{array}$ & $3 / 4 / 5$ & $6 / 19 / 48$ & 0.2127 \\
\hline Previous cytapheresis therapy (no. of patients with/without) & $6 / 6$ & $36 / 37$ & 0.9649 \\
\hline Previous biologics treatment (no. of patients with/without) & $0 / 12$ & $4 / 69$ & 0.4062 \\
\hline Previous AZA or 6-MP treatment (no. of patients with/without) & $1 / 11$ & $28 / 45$ & 0.0421 \\
\hline Hematocrit levels (mean \%) & 30.0 & 35.6 & 0.0038 \\
\hline White blood cell count $/ \mathrm{mm}^{3}$ (mean) & 9,633 & 9,441 & 0.8797 \\
\hline Lymphocyte count $/ \mathrm{mm}^{3}$ (mean) & 930 & 1,231 & 0.1387 \\
\hline Platelet count (mean; x103/.mm $)$ & 309 & 347 & 0.3979 \\
\hline Albumin levels (mean; g/dl) & 2.95 & 3.41 & 0.0256 \\
\hline C-reactive protein levels (mean; mg/dl) & 930 & 1,231 & 0.1387 \\
\hline O-PNI (mean) & 34.15 & 40.27 & 0.0069 \\
\hline C7-HRP (no. of patients with positive/negative C7-HRP) & $6 / 6$ & $17 / 56$ & 0.0536 \\
\hline PSL $\geq 10,000 \mathrm{mg}$ (no. of patients with/without PSL $\geq 10,000 \mathrm{mg}$ ) & $3 / 9$ & $9 / 64$ & 0.2427 \\
\hline
\end{tabular}

Left-sided colitis refers to patients who have mucosal lesions restricted to the rectum, sigmoid colon and descending colon. M, male; F, female; AZA, azathioprine; 6-MP, 6-mercaptopurine; O-PNI, onodera-prognostic nutritional index; C7-HRP, cytomegalovirus antigenemia; PSL, prednisolone.

a two-tailed Student's t-test. $\mathrm{P}<0.05$ was considered to indicate a statistically significant difference. Receiver operating characteristic (ROC) curves were plotted to compare the ability of each clinical variable to predict early colectomy. All statistical analyses were performed using StatView version 5.0 (SAS Institute, Cary, NC, USA), GraphPad Prism version 4.03 (GraphPad Software, Inc., San Diego, CA, USA) or SPSS version 16.0.1 (SPSS, Inc., Chicago, IL, USA).

\section{Results}

Factors affecting early colectomy. The comparison of patient clinical backgrounds between the early colectomy group and the non-early colectomy group is shown in Table II. The early colectomy group included significantly more patients who had severe disease activity (as defined by a score $>220$ in Seo's disease activity index) and an immunomodulator-naïve history (defined as patients without a history of immunomodulatiory medication). In addition, the early colectomy group had significantly lower hematocrit levels, serum albumin levels and O-PNI scores as compared with the colectomy-free group. However, no statistical difference was observed between the two groups in the presence of cytomegalovirus reactivation and the amount of prednisolone used prior to the initiation of CNI treatment.

Factors affecting overall colectomy. The comparison of patient clinical backgrounds between the overall colectomy group and the colectomy-free group is shown in Table III. C7-HRP

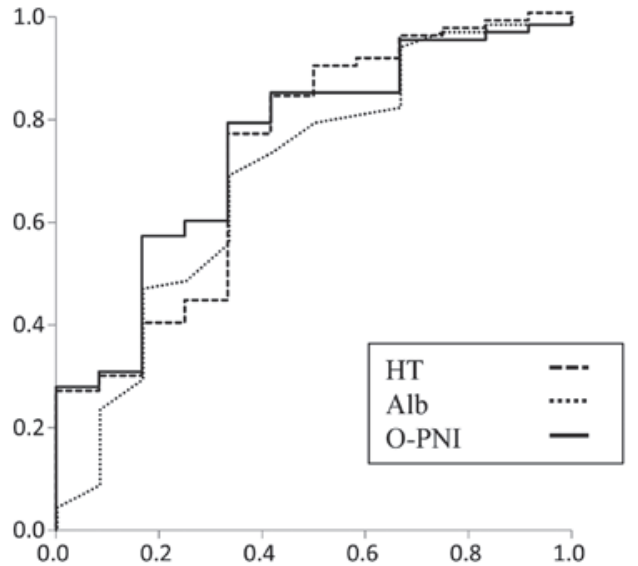

Figure 1. Receiver operating characteristic curves for factors predicting early colectomy. HT, dashed line; Alb, dotted line; O-PNI, solid line. Alb, albumin; HT, hematocrit; O-PNI, Onodera-prognostic nutritional index.

positivity and $>10,000 \mathrm{mg}$ prednisolone used prior to the initiation of CNI treatment were significant factors that predicted colectomy. However, there was no statistical difference between the two groups with regard to disease severity, the previous use of immunomodulators, hematocrit levels, serum albumin levels and O-PNI scores.

ROC analysis for factors affecting early colectomy. To evaluate the prognostic yield, ROC curves were plotted for 
Table III. Comparison of patient clinical backgrounds between the colectomy group and the non-colectomy group.

\begin{tabular}{|c|c|c|c|}
\hline Clinical characteristic & Colectomy group & Non-colectomy group & P-value \\
\hline Number of patients $(\mathrm{M} / \mathrm{F})$ & $34(19 / 15)$ & $51(33 / 18)$ & 0.4135 \\
\hline Age (mean; years) & 34.1 & 34.9 & 0.7701 \\
\hline Disease duration (mean; months) & 43.7 & 60.3 & 0.0519 \\
\hline Seo index (no. of patients <220/ $\geq 220$ ) & $18 / 16$ & $27 / 24$ & 1.0000 \\
\hline Disease severity (no. of patients with left-sided colitis/pancolitis) & $9 / 25$ & $20 / 31$ & 0.2247 \\
\hline Disease course (first attack/chronic continuous/relapse remitting) & 4/11/19 & $5 / 12 / 34$ & 0.5941 \\
\hline Previous cytapheresis therapy (no. of patients with/without) & $19 / 15$ & $23 / 28$ & 0.3299 \\
\hline Previous biologics treatment (no. of patients with/without) & $0 / 34$ & $4 / 47$ & 0.0944 \\
\hline Previous AZA or 6-MP treatment (no. of patients with/without) & $4 / 11 / 19$ & $5 / 12 / 34$ & 0.5941 \\
\hline Hematocrit (mean \%) & 33.5 & 35.8 & 0.1052 \\
\hline White blood cell count $/ \mathrm{mm}^{3}$ (mean) & 9,274 & 9,598 & 0.7191 \\
\hline Lymphocyte count $/ \mathrm{mm}^{3}$ (mean) & 1,223 & 1,166 & 0.6957 \\
\hline Platelet count (mean; $x 10^{3} / \mathrm{mm}^{3}$ ) & 326 & 351 & 0.4361 \\
\hline Albumin levels (mean; g/dl) & 3.30 & 3.37 & 0.6307 \\
\hline C-reactive protein levels (mean; mg/dl) & 2.53 & 2.83 & 0.7065 \\
\hline O-PNI (mean) & 39.14 & 39.57 & 0.7932 \\
\hline C7-HRP (no. of patients with positive/negative C7-HRP) & $14 / 20$ & $9 / 42$ & 0.0168 \\
\hline$P S L \geq 10,000 \mathrm{mg}$ (no. of patients with/without PSL $\geq 10,000 \mathrm{mg}$ ) & $8 / 26$ & $4 / 47$ & 0.0419 \\
\hline
\end{tabular}

Left-sided colitis refers to patients who have mucosal lesions restricted to the rectum, sigmoid colon and descending colon. M, male; F, female; AZA, azathioprine; 6-MP, 6-mercaptopurine; O-PNI, onodera-prognostic nutritional index; C7-HRP, cytomegalovirus antigenemia; PSL, prednisolone.

Table IV. Receiver operating characteristic analysis for factors affecting early colectomy.

\begin{tabular}{|c|c|c|c|c|c|c|c|}
\hline \multirow[b]{2}{*}{ Factor } & \multirow[b]{2}{*}{ AUC } & \multirow[b]{2}{*}{ P-value } & \multicolumn{2}{|c|}{$95 \%$ confidence interval } & \multirow[b]{2}{*}{ Sensitivity } & \multirow[b]{2}{*}{ Specificity } & \multirow[b]{2}{*}{ Cut-off value } \\
\hline & & & Lower & Upper & & & \\
\hline $\mathrm{Alb}(\mathrm{g} / \mathrm{dl})$ & 0.694 & 0.033 & 0.520 & 0.867 & 0.691 & 0.667 & 3.05 \\
\hline $\mathrm{HT}(\%)$ & 0.727 & 0.013 & 0.560 & 0.893 & 0.765 & 0.667 & 30.9 \\
\hline O-PNI & 0.749 & 0.006 & 0.600 & 0.898 & 0.794 & 0.667 & 34.1 \\
\hline
\end{tabular}

AUC, area under the curve; Alb, albumin; HT, hematocrit; O-PNI, onodera-prognostic nutritional index.

Table V. Hematocrit and O-PNI enhance the prediction of early colectomy.

\begin{tabular}{lcc}
\hline & \multicolumn{2}{c}{ O-PNI } \\
\cline { 2 - 3 } Rate of early colectomy & $\geq 34.1$ & $<34.1$ \\
\hline Hematocrit (\%) & & \\
$\geq 30.9$ & $4.0 \%(2 / 50)$ & $18.2 \%(2 / 11)$ \\
$<30.9$ & $16.7 \%(2 / 12)$ & $50.0 \%(6 / 12)$ \\
\hline
\end{tabular}

O-PNI, onodera-prognostic nutritional index.

early colectomy following CNI administration (Fig. 1). The area under the curve (AUC), the sensitivity and the specificity have been included in Table IV. The cut-off values were set for hematocrit and O-PNI using the ROC analyses. By combining the hematocrit and O-PNI, the patients were divided into four groups as shown in Table V. The early colectomy rate was 50\% in the group with the worst prognosis.

\section{Discussion}

In the present study, the predictors for early colectomy prior to the commencement of treatment with CNIs were analyzed. Notably, the results obtained were significantly different compared with the factors affecting overall colectomy. The significant factors predicting early colectomy were as follows: i) disease severity, ii) immunomodulator-naïve history, iii) lower serum hematocrit, iv) lower serum albumin levels and v) lower O-PNI scores. Conversely, the significant factors predicting the overall colectomy risk were i) C7-HRP positivity and ii) $>10,000 \mathrm{mg}$ prednisolone used prior to the 
initiation of CNI treatment; these results were concordant with those of our previous report (20).

In accordance with these results, previous studies demonstrated that disease severity, the use of immunomodulators, and hematocrit levels can affect the rate of colectomy in UC (17,29). Significantly lower serum albumin levels were observed in the early colectomy group. Serum albumin was included in the O-PNI score. In addition, O-PNI has better predictability for colectomy as compared to serum albumin levels alone in the present study. Lymphocyte count does not appear to be the only significant factor but has a positive effect in the prediction of early colectomy. The results of the present study suggested that blood tests can predict the prognosis for early colectomy.

We have previously reported that the factors affecting the rate of colectomy at 1 year and the overall colectomy rate were i) CNI non-responsiveness, ii) $>10,000 \mathrm{mg}$ prednisolone used prior to the initiation of CNI treatment and iii) positivity for C7-HRP (20). However, the response to CNIs may not be obvious prior to the start of CNI administration. Therefore, factors that could be assessed prior to CNI administration were prednisolone dosage and C-7HRP positivity. These two factors were significant for the prediction of overall colectomy but not for early colectomy in the present study. With regard to the dosage of prednisolone, certain patients underwent colectomy due to prednisolone-dependency and not disease severity. With regard to C7-HRP positivity, patients with cytomegalovirus reactivation who were treated with antiviral drugs and had once responded to CNIs tended to relapse during the earlier period and subsequently underwent colectomies.

Previous guidelines recommend that induction of a second-line treatment such as CNIs should be commenced soon after the determination of the steroid-refractory status $(16,30)$. However, in clinical settings, patients occasionally receive high-dose steroids for $>2$ weeks prior to being transferred to the Shiga University of Medical Science Hospital. In these cases, the serum albumin or hematocrit levels are frequently low. The results of the present study suggested that early induction of CNIs may lead to improved results. Furthermore, the prediction of poor prognosis prior to the commencement of treatment will prove useful in clinical practice situations. This would allow the intensity of treatment to be established and for a surgeon to be consulted in a suitable time-frame.

In conclusion, the results of the present study demonstrated that clinical variables such as hematocrit levels and O-PNI score determined prior to the commencement of treatment with CNIs were significant factors in predicting colectomy. These results could be used as a guide to predict the outcome of the patients in clinical settings.

\section{References}

1. Okamoto R and Watanabe M: Role of epithelial cells in the pathogenesis and treatment of inflammatory bowel disease. J Gastroenterol 51: 11-21, 2016.

2. Matsuoka $\mathrm{K}$ and Hibi T: Treatment guidelines in inflammatory bowel disease: the Japanese perspectives. Dig Dis 31: 363-367, 2013.

3. Ogata H, Matsui T, Nakamura M, Iida M, Takazoe M, Suzuki Y and Hibi T: A randomised dose finding study of oral tacrolimus (FK506) therapy in refractory ulcerative colitis. Gut 55: 1255-1262, 2006.
4. Lichtiger S, Present DH, Kornbluth A, Gelernt I, Bauer J, Galler G, Michelassi F and Hanauer S: Cyclosporine in severe ulcerative colitis refractory to steroid therapy. N Eng J Med 330: 1841-1845, 1994

5. Dignass A, Van Assche G,Lindsay JO, Lémann M, J, Colombel JF, Danese S, D'Hoore A, Gassull M, Gomollón F, et al; European Crohn's and Colitis Organisation (ECCO): The second European evidence-based Consensus on the diagnosis and management of Crohn's disease: Current management. J Crohns Colitis 4: 28-62, 2010.

6. Chang KH, Burke JP and Coffey JC: Infliximab versus cyclosporine As rescue therapy in acute severe steroid-refractory ulcerative colitis: A systematic review and meta-analysis. Int J Colorectal Dis 28: 287-293, 2013.

7. Laharie D, Bourreille A, Branche J, Allez M, Bouhnik Y, Filippi J, Zerbib F, Savoye G, Nachury M, Moreau J, et al: Ciclosporin versus infliximab in patients with severe ulcerative colitis refractory to intravenous steroids: A parallel, open-label randomised controlled trial. Lancet 380: 1909-1915, 2012

8. Hamilton MJ, Snapper SB and Blumberg RS: Update on biologic pathways in inflammatory bowel disease and their therapeutic relevance. J Gastroenterol 47: 1-8, 2012.

9. Kornbluth A and Sachar DB; Practice Parameters Committee of the American College of Gastroenterology: Ulcerative colitis practice guidelines in adults: American College Of Gastroenterology, Practice Parameters Committee. Am J Gastroenterol 105: 501-523, 2010.

10. Sjöberg M, Walch A, Meshkat M, Gustavsson A, Järnerot G, Vogelsang H, Hertervig E, Novacek G, Friis-Liby I, Blomquist L, et al: Infliximab or cyclosporine As rescue therapy in hospitalized patients with steroid-refractory ulcerative colitis: A retrospective observational study. Inflamm Bowel Dis 18: 212-218, 2012.

11. Dean KE, Hikaka J, Huakau JT and Walmsley RS: Infliximab or cyclosporine for acute severe ulcerative colitis: A retrospective analysis. J Gastroenterol Hepato 27: 487-492, 2012.

12. Nakase H, Matsumura K, Yoshino T and Chiba T: Systematic review: Cytomegalovirus infection in inflammatory bowel disease. J Gastroenterol 43: 735-740, 2008.

13. Criscuoli V, Mocciaro F, Orlando A, Rizzuto MR, Renda MC and Cottone M: Cytomegalovirus disappearance after treatment for refractory ulcerative colitis in 2 patients treated with infliximab and 1 patient with leukapheresis. Inflamm Bowel Dis 15: 810-811, 2009.

14. D'Ovidio V, Vernia P, Gentile G, Capobianchi A, Marcheggiano A, Viscido A, Martino P and Caprilli R: Cytomegalovirus infection in inflammatory bowel disease patients undergoing anti-TNFalpha therapy. J Clin Virol 43: 180-183, 2008.

15. Nakase $\mathrm{H}$ and Chiba T: TNF-alpha is an important pathogenic factor contributing to reactivation of cytomegalovirus in inflamed mucosa of colon in patients with ulcerative colitis: Lesson from clinical experience. Inflamm Bowel Dis 16: 550-551, 2010.

16. Dignass A, Lindsay JO, Sturm A, Windsor A, Colombel JF, Allez M, D'Haens G, D'Hoore A, Mantzaris G, Novacek G, et al: Second European evidence-based consensus on the diagnosis and management of ulcerative colitis part 2: Current management. J Crohns Colitis 6: 991-1030, 2012.

17. Reinisch W, Reinink AR and Higgins PD: Factors associated with poor outcomes in adults with newly diagnosed ulcerative colitis. Clin Gastroenterol Hepatol 13: 635-642, 2015.

18. Monstad I, Hovde O, Solberg IC and A Moum B: Clinical course and prognosis in ulcerative colitis: Results from population-based and observational studies. Ann Gastroentero 27: 95-104, 2014.

19. Saito K, Katsuno T, Nakagawa T, Saito M, Sazuka S, Sato T, Matsumura T, Arai M, Miyauchi H, Matsubara $\mathrm{H}$ and Yokosuka O: Predictive factors of response to intravenous ciclosporin in severe ulcerative colitis: The development of a novel prediction formula. Aliment Pharmacol Ther 36: 744-754, 2012.

20. Bamba S, Andoh A, Imaeda H, Ban H, Kobori A, Mochizuki Y, Shioya M, Nishimura T, Inatomi O, Sasaki M, et al: Prognostic factors for colectomy in refractory ulcerative colitis treated with calcineurin inhibitors. Exp Ther Med 4: 99-104, 2012.

21. Onodera T, Goseki N and Nosaki G: Prognostic nutritional index in gastrointestinal surgery of malnourished cancer patients. Nihon Geka Gakkai Zasshi 85: 1001-1005, 1984 (In Japanese).

22. Goseki N, Okamoto A and Onodera T: Postoperative nutritional assessment in gastric and colorectal cancer. Nihon Geka Gakkai Zasshi 87: 853-838, 1986 (In Japanese). 
23. Yagi T, Yamagishi F, Sasaki Y, Itakura M, Fujikawa A, Kuga M and Ishimaru G: A study on cases developed pulmonary tuberculosis after receiving gastrectomy. Kekkaku 79: 355-359, 2004 (In Japanese).

24. Sagawa M, Katsube T, Konno S, Murayama M, Yamaguchi K, Isohata N, Yoshimatsu K, Shiozawa S, Shimakawa T, Naritaka Y and Ogawa K: The significance of Onodera's prognostic nutritional index for the treatment of gastrointestinal cancer. Gan To Kagaku Ryoho 35: 2253-2255, 2008 (In Japanese).

25. Fukushima K, Ueno Y, Kawagishi N, Kondo Y, Inoue J, Kakazu E, Ninomiya M, Wakui Y, Saito N, Satomi S and Shimosegawa T: The nutritional index 'conut' is useful for predicting long-term prognosis of patients with end-stage liver diseases. Tohoku J Exp Med 224: 215-219, 2011.

26. Kang SH, Cho KH, Park JW, Yoon KW and Do JY: Onodera's prognostic nutritional index as a risk factor for mortality in peritoneal dialysis patients. J Korean Med Sci 27: 1354-1358, 2012.
27. Bamba S, Tsujikawa T, Inatomi O, Nakahara T, Koizumi Y, Saitoh Y, Sasaki M, Fujiyama Y and Andoh A: Factors affecting the efficacy of cyclosporin a therapy for refractory ulcerative colitis. J Gastroenterol Hepatol 25: 494-498, 2010.

28. Seo M, Okada M, Yao T, Ueki M, Arima S and Okumura M An index of disease activity in patients with ulcerative colitis. Am J Gastroenterol 87: 971-976, 1992.

29. Kaplan GG, Seow CH, Ghosh S, Molodecky N, Rezaie A, Moran GW, Proulx MC, Hubbard J, MacLean A, Buie D and Panaccione R: Decreasing colectomy rates for ulcerative colitis: A population-based time trend study. Am J Gastroenterol 107: 1879-1887, 2012.

30. Mowat C, Cole A, Windsor A, Ahmad T, Arnott I, Driscoll R, Mitton S, Orchard T, Rutter M, Younge L, et al: Guidelines for the management of inflammatory bowel disease in adults. Gut 60: 571-607, 2011. 